\title{
Shear Wave Elastography
}

In the last years, liver elastography became an important target for many research groups, which tried to establish the real value of this new method, especially for the evaluation of diffuse hepatopathies. Liver biopsy was considered as the "gold standard" for liver fibrosis assessment and the first elastographic method, transient elastography, was compared to the morphologic evaluation (liver biopsy). Later studies, evaluating new elastographic methods, tried to demonstrate their non-inferiority as compared to this validated method.

In the last years we observed a dramatic decrease in the number of liver biopsies performed for liver fibrosis assessment in chronic hepatitis, as a result of increasing use of the non-invasive modalities, especially in Europe. Biological tests (direct or indirect) and elastographic methods for liver stiffness evaluation became more popular and are used for prognosis assessment, for decision regarding treatment and for the follow-up of patients with chronic hepatopathies.

Tissue elasticity can be described qualitatively by evaluating the relative displacements caused by static or dynamic deformations. Quantitative techniques estimate the deformation rate, which indirectly characterize environmental stiffness.

The first elastographic method used in clinical practice was transient elastography (TE). Now, after more than 10 years experience with this method, several metaanalyses showed its real value. The weak points of this method are: It is a quite blind method (no real time ultrasound use); it is not feasible in patients with ascites and in approximately $15-20 \%$ of patients, reliable measurements cannot be obtained.
More recently, shear wave elastography (SWE) was developed. Quantitative elastography techniques provide high resolution information about tissue elasticity in a region of interest. To obtain a quantitative elastographic assessment, shear waves are generated into the tissue, then tracked and measured. Complex equipment is used to generate them and to make high-resolution measurements of their propagation velocity. Several techniques can be used to generate the shear waves:

Acoustic Radiation Force Impulse (ARFI) Elastography

The acoustic radiation force causes tissue displacement centered on a region of interest. These displacements propagate through the tissue in the form of shear waves and an ultrasound system is used to follow the shear waves' propagation. The time scale of tissue's response is much slower than that of the ultrasonic wave propagation. ARFI elastography is integrated into an Acuson S2000 ultrasound machine, from Siemens.

Principle of ARFI Elastography: A short duration, high-intensity acoustic "push pulse" is transmitted by the transducer, followed by a series of diagnostic intensity pulses, which are used to track the tissue displacement caused by the push pulse. The tissue response to the radiation force is observed using conventional Bmode imaging pulses, and it is possible to measure and display the quantitative shear-wave velocity (meters/second $\mathrm{m} / \mathrm{s}$ ) from the ARFI generated displacements. This velocity is proportional to the square root of the tissue's elasticity modulus. Because the shear wave velocity depends on tissue stiffness, it is possible to 
apply ARFI technology to elastography. This technology was named "Virtual Touch Tissue Quantification". Soft tissues, being elastic, will deform more than rigid tissue, whose elasticity is much lower. The deformation associated with high intensity ultrasonic pulse propagation is followed by a relaxation process, after which the tissue returns to its original configuration.

In the final phase, the region is scanned with a normal intensity (diagnostic) US beam and a new B-mode image is acquired. By comparing it with the reference image, displacements occurring in different areas can be calculated.

Supersonic Shear Imaging (SSI) Technique (Aixplorer system) relies on the simultaneous use of both ultrasound and shear waves, to better characterize and quantify tissue stiffness.

To produce shear waves of sufficient amplitude in the entire region of interest, it generates a sequence of stimulation pulses focused at different depths. The pushing pulses form a pushing beam. The rapid change in focal depth in the pushing beam with time is equivalent to a high output source moving rapidly through the tissue. Where the source speed is greater than the wave speed, the source is said to be moving at supersonic speed, hence the term 'supersonic imaging'.

The Aixplorer system can generate, capture and quantify shear waves velocity, by acquiring data up to 200 times faster than conventional ultrasound technology. Shear wave velocity is directly related to the quantifiable measurement of tissue elasticity. The Aixplorer system generates in real-time a quantitative color coded map displaying local tissue elasticity for a large region of interest. An adjustable color scale indicates tissue elasticity ranging from soft (blue) to hard (red). In the same time, an area of interest can be chosen for which the elasticity is displayed in kiloPascals ( $\mathrm{kPa}$ ) or in $\mathrm{m} / \mathrm{s}$.

No compression is needed to obtain the real-rime shear wave elastography map of the tissue, which depicts the tissue elasticity (stiffness) by color. The tissue elasticity is calculated based on shear wave propagation speed into the tissue. Based on Young's modulus, if shear wave propagation speed can be measured, the tissue elasticity can be determined. Not only local measurements of tissue elasticity can be obtained on a two-dimensional map, but also, with the Q-Box, every pixel in a region of interest can be measured.
Clinical results with ARFI elastography show that this method is reliable for liver stiffness assessment as a marker of fibrosis in patients with chronic hepatitis (especially HCV, better than HBV), with promising results in patients with NAFLD, and in post-transplant patients. On the other hand, many publications evaluated this method for the prediction of portal hypertension or of liver cirrhosis complications (sometimes using also spleen stiffness measurements). Published studies demonstrated similar accuracy for TE and ARFI for the non-invasive assessment of liver fibrosis. Also, it was demonstrated that elevated aminotransferase levels increase liver stiffness values assessed by ARFI and that a better correlation of ARFI measurements with histological liver fibrosis exists when quality indicators (IQR $<30 \%$ and $\mathrm{SR}>60 \%)$ are used. Interobserver variability of this method was reported to be good.

Concerning SSI, this shear wave elastographic method is only at the beginning and only few publications are available, but it shows promising results for the clinical practice.

The main advantages of shear wave elastography techniques are: They are included in an ultrasound machine, elastography being performed during the ultrasound evaluation (real time techniques); they can be performed in patients with as- cites; they have a lower rate of invalid measurements (as compared to TE).

In conclusion, shear wave elastography seems to be a new step for a real time noninvasive liver stiffness assessment.

\section{Prof. Ioan Sporea, $M D, P h D$}

Department of Gastroenterology and Hepatology, "Victor Babeș" University of Medicine and Pharmacy Timișoara, Romania, WFUMB Center of Excellence (COE)

\section{Assoc. Prof. Ioan Lie, PhD}

Applied Electronics Department, Electronics and Telecomunications Faculty, "Politehnica” University Timișoara, Romania

\section{Selective references}

1 Tsochatzis EA, Gurusamy KS, Ntaoula S, et al. Elastography for the diagnosis of severity of fibrosis in chronic liver disease: a meta-analysis of diagnostic accuracy. J Hepatol 2011; 54: 650-659

2 Friedrich-Rust M, Nierhoff J, Lupșor M, et al. Performance of Acoustic Radiation Force Impulse imaging for the staging of liver fibrosis: a pooled meta-analysis. J Viral Hepat 2012; 19: e212-e219

3 Bavu E, Gennisson J-L, Couarde M et al. Noninvasive in vivo liver evaluation using supersonic shear imaging: a clinical study on 113 hepatitis $C$ virus patients. Ultrasound Med Biol 2011; 37: 1361-1373

4 Virtual Touch - White paper

5 Supersonic Imagine - Technology Brochure

6 Sporea I, Șirli R et al. Hepatic Elastography using ultrasound waves. E-book (in press) 